\title{
Análisis factorial de la escala de actitudes hacia el alumnado con discapacidad en educación física (EAADEF)
}

\author{
María del Carmen ÍÑIGUEZ-SANTIAGO ${ }^{1}$, Roberto FERRIZ ${ }^{2}$, \\ María Celestina MARTÍNEZ-GALINDO ${ }^{1}$, Marta María CEBRIÁN-SÁNCHEZ ${ }^{1}$ \\ y Raúl REINA ${ }^{1}$. \\ ${ }^{1}$ Universidad Miguel Hernández de Elche. España \\ ${ }^{2}$ Universidad CEU Cardenal Herrera. España
}

(Recibido, 16 marzo 2017; Aceptado, 16 junio 2017)

RESUMEN: La inclusión de estudiantes con discapacidad en educación física (EF) está influida directamente por la actitud que los compañeros presentan al respecto, siendo necesario contar con herramientas válidas y fiables para evaluar dicha actitud. El objetivo de este estudio fue crear y analizar las propiedades psicométricas de un instrumento para medir la actitud hacia la inclusión del alumnado con discapacidad en las clases de EF. Participaron 609 estudiantes de EF, de 14 a 19 años. Se realizaron cuatro análisis factoriales exploratorios (AFE) y un análisis factorial confirmatorio (AFC). Se analizaron las propiedades psicométricas de la escala (componente conductual y cognitivo) y su relación con cuatro variables socio-demográficas. La fiabilidad del componente cognitivo estuvo por debajo de lo recomendado y el AFE no apoyó un modelo formado por dos dimensiones de la actitud. Se obtuvo una medida unidimensional con cuatro ítems del componente conductual. La Escala de Actitudes del Alumnado hacia la Discapacidad en Educación Física (EAADEF) reveló excelentes índices de ajuste en el AFC, y se reveló invariante respecto a las variables socio-demográficas. También se encontraron valores altos de consistencia interna de los ítems y del constructo de actitud ( $\geq .77)$. Esta escala ofrece una nueva solución a algunas limitaciones reveladas en instrumentos de medida anteriores.

Palabras clave: Inclusión, necesidades especiales, sensibilización, educación física inclusiva.

Factorial analysis of the attitudes' scale towards students with disabilities in physical education (ASSDPE)

ABSTRACT: The inclusion of students with disabilities in physical education (PE) is directly influenced by the peers' attitudes, which requires valid and reliable tools to measure this attitude. The aim of this study was to create and to analyse the psychometric properties of an instrument to measure attitudes towards the inclusion of students with disabilities in the PE classes. This study involved 609 PE students from 14 to 19 years old. Four exploratory factor analysis (EFA) and one confirmatory factor analysis (CFA) were conducted. We analysed the psychometric properties of the scale (cognitive and behavioural components) and its relation with four socio-demographic variables. The reliability of the cognitive component was below that recommended and the EFA didn't support a model formed by two dimensions of the attitude. A one-dimensional measure with four elements of the behavioural component was 
obtained. The Scale of Attitudes towards Students with Disabilities in Physical Education (SASDPE) revealed excellent adjustment indexes in the CFA, being invariant with respect to the socio-demographic variables. High values of internal consistency of the items and for the attitude construct ( $\geq .77)$ were also found. This scale offers new solutions to some limitations revealed in previous instruments to evaluate attitudes towards disability in PE.

Keywords: Inclusion, special needs, awareness, inclusive physical education.

Correspondencia: ${ }^{1}$ Raúl Reina Vaíllo. Universidad Miguel Hernández de Elche, Centro de Investigación del Deporte. Avda. de la Universidad s/n, 03202, Elche. Email: rreina@goumh.es

\section{Introducción}

El establecimiento de estrategias educativas para fomentar la inclusión de estudiantes con discapacidad en las aulas permite su aceptación por parte de sus iguales sin discapacidad, propiciándose relaciones positivas (Vignes, Coley, Grandjean, Godeau, y Arnaud, 2008). Resulta pues necesario programar e implementar acciones concretas y continuadas para lograr una plena aceptación e inclusión (Aguado, Alcedo, y Arias, 2008). Según Lindsay y Edwards (2013), los programas educativos (e.g., simulaciones, intervenciones curriculares, contacto con personas con discapacidad), orientados a provocar un cambio de actitud y la aceptación de estudiantes con discapacidad, pueden romper con los estereotipos y advertir acerca de las barreras existentes para con su inclusión.

Para lograr una educación inclusiva, diferentes investigadores (e.g., Flórez, Aguado, y Alcedo, 2009) han reconocido la importancia que tienen las actitudes mostradas por el alumnado sin discapacidad y el profesorado, así como los contenidos impartidos, debiendo estar éstos adaptados al alumnado con discapacidad en cada aula y contexto. Según Campos, Ferreira y Block (2014), de todas las áreas del currículum, son las clases de educación física (EF) las que, por sus características curriculares y estructurales, propician una mayor interacción entre pares con y sin discapacidad, fomentando una participación activa y efectiva de todo el alumnado (Ocete, 2016).

Para Triandis (1971), la actitud es una idea provista de una carga emocional que predispone a poder realizar una serie de acciones ante un determinado tipo de situaciones sociales. Este autor propuso una concepción de la actitud dividida en tres componentes: la idea (componente cognitivo), la emoción asociada a la idea (componente afectivo), y la predisposición a la acción (componente conductual). Vignes et al. (2008) realizaron en diferentes disciplinas una revisión de los instrumentos que medían la actitud bajo este paradigma (educación, psicología, epidemiología, rehabilitación y pediatría). De los 19 instrumentos analizados, tanto cuantitativos como cualitativos, dos medían los tres

componentes de la actitud, uno los componentes cognitivo y comportamental, y 16 un único componente (cuatro el afectivo, cinco el conductual, y siete el cognitivo). De los resultados encontrados se desprende la necesidad de discutir sobre la idoneidad del modelo conceptual y tridimensional de la actitud propuesto por Triandis (1971). Así, debido a la proliferación de proyectos educativos que están permitiendo avanzar de la teorización de modelos, estrategias y protocolos, a la aplicación práctica de las teorías inclusivas en EF en contextos reales (Reina, Hutzler, Íniguez-Santiago, y Moreno-Murcia, 2016), es preciso contar con instrumentos de medida válidos y fiables (Lindsay y Edwards, 2013). 
En el contexto de las clases de EF, son escasos los instrumentos que se han elaborado para medir la actitud, especialmente en español. Ocete (2016) adaptó al contexto español el Children's Attitudes Towards Integrated Physical Education-Revised (CAIPE-R: Block, 1995), recientemente validado por Cordente-Mesas, González-Víllora, Block, y ContrerasJordán (2016) y denominado Children's Attitudes towards Integrated Physical EducationSpanish version (CAIPE-SP). Aunque el CAIPE se presenta como una herramienta apropiada para EF (Campos et al., 2014), las versiones existentes no disponen, por ejemplo, de análisis de invarianza de diferentes variables demográficas como se aborda en el presente estudio.

Reina et al. (2016) elaboraron la Attitudes Towards Inclusion of Students with Disabilities in Physical Education Scale (AISDPE), incorporando una definición del concepto de discapacidad como objeto social de referencia para poder responder a los ítems del cuestionario. La AISDPE presentó una versión inicial formada por 32 ítems (18 para el componente cognitivo, ocho para el conductual y seis para el afectivo). Los análisis factoriales exploratorios (AFE) y el confirmatorio (AFC) apoyaron una versión con dos de los tres componentes de la actitud: cognitivo (siete ítems) y conductual (10 ítems). El cuestionario ofreció unos valores aceptables de consistencia interna (alfa de Cronbach $\geq .74$ ), así como una correlación moderada entre ambos constructos. Sin embargo, no se realizaron suficientes análisis para determinar su validez (e.g., análisis de invarianza respecto al género).

Los instrumentos de medida disponibles en la actualidad para analizar la actitud hacia la inclusión de personas con discapacidad en el contexto educativo, podrían evolucionar a fin de ofrecer mejores evidencias de validez y fiabilidad (Flórez et al., 2009). Esta necesidad que transciende al contexto de EF (e.g., no se conoce si el AISDPE permite relacionar la actitud con el género o el haber tenido contacto previo con personas con discapacidad), se acentúa si se tiene en cuenta el escaso número de herramientas de las que se dispone para medir la actitud.

Identificada esta necesidad en la bibliografía, el objetivo de este estudio fue desarrollar una escala para medir la actitud hacia el alumnado con discapacidad en EF. En primer lugar, se analizaron las propiedades psicométricas de un modelo hipotetizado de dos subescalas, que consideró los componentes cognitivo y conductual del modelo de tres componentes de las actitudes (Reina et al., 2016). En segundo lugar, se analizó la invarianza para cuatro variables socio-demográficas que podrían ser relevantes para evaluar las actitudes del alumnado hacia sus compañeros con discapacidad: a) género, b) tener contacto con un familiar o amigo con discapacidad, c) tener contacto con un compañero con discapacidad en EF y d) haber participado previamente en una actividad deportiva con personas con discapacidad. En tercer lugar, se calculó la correlación entre los ítems, evaluando la consistencia interna y de constructo del instrumento.

\section{Método}

\section{Participantes}

Participaron 609 estudiantes (319 chicas y 290 chicos) de EF, con edades comprendidas entre los 14 y 19 años (15.66 \pm 1.07 años). El alumnado pertenecía a cinco centros educativos de tres localidades españolas, y se encontraban cursando $3^{\circ}$ y $4^{\circ}$ de educación secundaria obligatoria, así como $1^{\circ}$ de bachillerato (educación secundaria post-obligatoria). El alumnado recibía dos sesiones a la semana de $\mathrm{EF}$, con una duración de 55 minutos cada una de ellas. 


\section{Medidas}

Escala de Actitud hacia el Alumnado con Discapacidad en Educación Física (EAADEF). Se incluyeron ocho ítems de la EAADEF, cuatro por factor (ver Anexo), para el componente conductual (e.g., "prefiero no relacionarme con personas con discapacidad") y cognitivo (e.g., "las personas con ceguera siempre han de llevar un guía que los acompañe de un lado para otro") del modelo de tres componentes de las actitudes (Triandis, 1971). Se empleó una escala Likert de 1 (totalmente en desacuerdo) a 5 (totalmente de acuerdo) y la sentencia previa: "En educación física, con respecto a las personas con discapacidad...". Puntuaciones más altas en la escala indican una actitud menos inclusiva o facilitadora de la participación del alumnado con discapacidad en EF.

Variables socio-demográficas. Se incluyeron cuatro variables respecto al: a) género

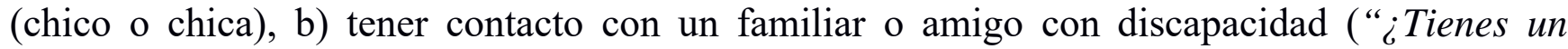
familiar o amigo/a cercano con discapacidad?"), c) tener contacto con un compañero con discapacidad en EF ( “ ¿Has tenido alguna vez un compañero/a con discapacidad en tu clase de educación física?”) y d) haber participado previamente en una actividad deportiva con personas con discapacidad (" ¿Has participado en alguna actividad deportiva con personas con discapacidad?”). Para la pregunta respecto al género los participantes marcaban con una equis la opción correspondiente, mientras que para el resto de preguntas debían responder marcando entre dos opciones de respuesta: "Sí" o "No".

\section{Procedimiento}

Después de una revisión de la literatura científica, cuatro expertos en el ámbito de la discapacidad y de las ciencias de la actividad física y del deporte, redactaron ocho ítems (cuatro del componente conductual y cuatro del cognitivo) para medir las actitudes hacia la discapacidad en EF. La elaboración de los ítems se apoyó en el modelo de actitud de Triandis (1971) y otras escalas previas (e.g., Bossaert y Petry, 2013; De Boer et al., 2012; Reina et al., 2016). Seguidamente, para evaluar la validez de contenido, los ítems fueron revisados por dos expertos ajenos. Para la inclusión, exclusión o modificación de los ítems elaborados, se tuvieron en cuenta las siguientes condiciones: (a) incluir aquellos ítems donde hubiera un 100\% de coincidencia favorable entre los expertos; (b) revisar y reformular los ítems para los que no se alcanzara un consenso del 100\%; y (c) excluir y sustituir por un nuevo ítem aquellos donde no hubiera una coincidencia del 100\%. Después de su evaluación, ambos expertos indicaron un $100 \%$ de consenso para incluir los ocho ítems, sin necesidad de ninguna modificación. Finalmente, los ítems fueron intercalados en la EAADEF para su administración.

El muestreo no fue aleatorio, pues los cinco centros educativos que participaron fueron seleccionados atendiendo a la proximidad geográfica y voluntariedad de participar. Todo el procedimiento seguido, hasta lograr la validación de la EAADEF, siguió las directrices éticas de la American Psychological Association (2010).

En la parte superior del cuestionario se añadió la siguiente explicación del término discapacidad: "una persona con discapacidad es aquella, que debido a la alteración de una estructura o función corporal (física, sensorial o intelectual), tiene limitaciones a la hora de realizar las actividades que serían normales”. 


\section{Análisis de datos}

Para analizar las propiedades psicométricas del cuestionario se realizaron los AFE y un AFC. Siguiendo las recomendaciones de Brown (2006), la muestra total fue dividida de forma aleatoria en dos subgrupos. Con la primera submuestra de 311 estudiantes (162 chicas y 149 chicos) se realizaron AFE, mientras que la segunda submuestra de 298 estudiantes (157 chicas y 141 chicos) se empleó para un AFC y el resto de análisis del estudio.

En primer lugar, para comprobar si los ítems seleccionados se agrupaban en dos factores independientes, se realizaron AFE mediante el método de extracción por componentes principales (sin fijar el número de factores a extraer) y rotación oblicua (promax), dado que podían obtenerse factores correlacionados de manera moderada (Brown, 2006). Para comprobar la idoneidad de aplicar el AFE se empleó el estadístico Kaiser-Meyer-Olkin (KMO) y la prueba de esfericidad de Bartlett.

En segundo lugar, para testar el modelo factorial propuesto, se realizó un AFC. Para este modelo, dado que el coeficiente de Mardia reveló que no se cumplió la distribución de normalidad (media normalizada $=14.85$ ), se utilizó el método de máxima verosimilitud junto con el procedimiento de bootstrapping. Los estimadores no se vieron afectados por la falta de normalidad, por lo que fueron considerados suficientemente robustos (Byrne, 2001). Para analizar la bondad de ajuste se utilizó un conjunto de varios índices: ratio entre chi cuadrado y grados de libertad $\left(\chi^{2} / g l\right)$, CFI (Comparative Fit Index), IFI (Incremental Fit Index), RMSEA (Root Mean Square Error of Approximation) y SRMR (Standardized Root Mean Square Residual). Dado que el $\chi^{2}$ es muy sensible al tamaño muestral (Jöreskog y Sörbom, 1993), se empleó el $\chi^{2} / g l$, para el que se consideran aceptables valores inferiores a 3 (Schermelleh-Engel, Moosbrugger, y Müller, 2003). Por su parte, los índices incrementales (i.e., CFI e IFI) mostrarían un ajuste aceptable con valores iguales o superiores a .95, mientras que para los índices de error se consideran aceptables valores iguales $\mathrm{o}$ inferiores a .06 para el RMSEA y .08 para el SRMR (Hu y Bentler, 1999).

En tercer lugar, se comprobó la invarianza de la estructura factorial con respecto a las cuatro variables socio-demográficas, requiriendo la ausencia de diferencias significativas entre el modelo sin restricciones (Modelo 1) y el modelo con pesos de medida invariantes (Modelo 2) (Byrne, Shavelson, y Multen, 1989). Además, se calcularon los estadísticos descriptivos, correlaciones bivariadas entre los ítems y consistencia interna de la escala (alfa de Cronbach $=\alpha$ ) y del constructo (índice Omega $=\omega$ ). Se utilizaron los paquetes estadísticos Statistical Package for Social Sciences (versión 21.0 para Windows, SPSS Inc, Chicago, IL, USA) para los AFE, y AMOS 20.0 para el AFC.

\section{Resultados}

\section{Análisis factoriales exploratorios, análisis de consistencia interna de la escala y del constructo}

Se realizó un primer AFE para comprobar si los ítems creados para los componentes conductual y cognitivo de la actitud se agrupaban en dos factores. La medida de adecuación muestral de KMO (.76) y la prueba de esfericidad de Bartlett $\left(\chi^{2}=471.74 ; g l=28 ; p<.001\right)$ indicaron la posibilidad de efectuar este análisis. El AFE identificó dos factores (ver Tabla 1), obteniendo todos los ítems valores de saturación iguales o por encima de .50 en su factor 
correspondiente, a excepción del ítem 6 del componente cognitivo, que no llegó a saturar de acuerdo al valor mínimo recomendado ( $\geq$.40) (Nunnally, 1978).

Tabla1. Análisis Factorial Exploratorio Inicial de la EAADEF.

\begin{tabular}{l|c|c}
\hline \multirow{2}{*}{ Ítems } & \multicolumn{2}{|c}{ Factores } \\
\cline { 2 - 3 } & 1 & 2 \\
\hline Ítem 1 & .78 & -.05 \\
Ítem 3 & .73 & .02 \\
Ítem 5 & .81 & -.05 \\
Ítem 7 & .79 & .05 \\
Ítem 2 & .22 & .51 \\
Ítem 4 & -.06 & .72 \\
Ítem 6 & .27 & .38 \\
Ítem 8 & -.15 & .74 \\
Varianza explicada & $34.65 \%$ & $15.81 \%$ \\
Varianza total & $50.46 \%$ & \\
\hline
\end{tabular}

Nota. Factor $1=$ Componente conductual; Factor $2=$ Componente cognitivo

Debido al bajo valor de saturación obtenido para el ítem 6, un segundo AFE fue realizado excluyendo este ítem. El AFE mantuvo los dos factores iniciales, obteniendo todos los ítems valores de saturación entre .51 y .81 en su factor correspondiente. El componente conductual (37.63\%) y el cognitivo (17.76\%) explicaron un $55.39 \%$ de la varianza total. No obstante, aunque el componente conductual presentó un valor adecuado de consistencia interna $(\alpha=.79)$ y de fiabilidad de constructo $(\omega=.78)$, el componente cognitivo mostró un valor para ambos indicadores de fiabilidad por debajo de lo recomendado (.41 y .56 , respectivamente).

Debido a la baja fiabilidad del componente cognitivo, se realizó un tercer AFE forzando el análisis para la extracción de un solo factor, que agrupara los componentes conductual y cognitivo con el objetivo de aumentar el valor de fiabilidad de la escala, explicando así un $37.63 \%$ del total de la varianza. Sin embargo, los tres ítems del componente cognitivo obtuvieron valores de saturación entre .21 y .46, mientras que los del factor conductual presentaron valores que oscilaron entre .79 y .75. Así, la posibilidad de un cuestionario de dos factores fue descartada, pasando a testar un modelo factorial que considerara solamente el componente conductual.

Como se puede observar en la Tabla 2, el cuarto AFE reveló unos valores de saturación para los ítems de .75 o superiores, y una varianza explicada del $61.23 \%$. Debido a los buenos valores de saturación para todos los ítems, a que se obtuvo el porcentaje de varianza explicada más alto de todos los AFE realizados y que además los valores de fiabilidad de los ítems $(\alpha=$ .79) y del constructo conductual $(\omega=.78)$ fueron buenos, se eligió este modelo unidimensional de cuatro ítems para ser testado en un AFC con la segunda submuestra de 298 estudiantes. 
Tabla 2. Análisis Factorial Exploratorio de los ítems de la EAADEF.

\begin{tabular}{lc}
\hline Ítems & Factor \\
\hline Ítem 1 & .79 \\
Ítem 3 & .75 \\
Ítem 5 & .78 \\
Ítem 7 & .81 \\
\hline Varianza explicada & $61.23 \%$ \\
\hline
\end{tabular}

\section{Análisis factorial confirmatorio}

Se realizó un AFC (Tabla 3) para testar la estructura factorial de un único factor de cuatro ítems del componente conductual, obteniendo unos índices de ajuste excelentes: $\chi^{2}$ $(2, N=298)=2.908, p<.001 ; \chi^{2} / g l=1.45 ; \mathrm{CFI}=.99 ; \mathrm{IFI}=.99 ; \mathrm{RMSEA}=.039 ;$ SRMR $=$ .018 . Los pesos de regresión estandarizados obtenidos para los ítems fueron $\geq .57$.

Tabla 3. Análisis factorial confirmatorio de la EAADEF.

\begin{tabular}{lcc}
\hline Ítems & Pesos de regresión estandarizados & Varianzas residuales \\
\hline Ítem 1 & .63 & .68 \\
Ítem 3 & .77 & .45 \\
Ítem 5 & .74 & .40 \\
Ítem 7 & .57 & .60 \\
\hline
\end{tabular}

\section{Análisis de invarianza}

Los índices de ajuste para los modelos de invarianza multigrupo analizados en función de las cuatro variables socio-demográficas (Tabla 4) no revelaron diferencias significativas en el estadístico $\chi^{2}$ entre el Modelo 1 y el Modelo 2.

Tabla 4. Análisis de Invarianza por Género, Familiar o Amigo con Discapacidad, Compañero de EF con Discapacidad, Participación en Actividad Deportiva con Persona con Discapacidad.

\begin{tabular}{lcccccccc}
\hline \multicolumn{7}{c}{ Invarianza por género } \\
\hline Modelos & $\chi^{2}$ & $l$ & $g l$ & $\Delta \chi^{2}$ & $\Delta g l$ & CFI & IFI & RMSEA \\
\hline Modelo 1 & 3.76 & 4 & .94 & - & - & .99 & .99 & .000 \\
Modelo 2 & 5.37 & 4 & .77 & 1.61 & 3 & .99 & .99 & .000 \\
Modelo 3 & 21.22 & 8 & 2.65 & $17.46^{* *}$ & 4 & .95 & .95 & .075 \\
Modelo 4 & 50.70 & 12 & 4.23 & $46.95^{* * *}$ & 8 & .84 & .84 & .104 \\
\hline
\end{tabular}

Invarianza por contacto con familiar o amigo con discapacidad

\begin{tabular}{lcccccccc}
\hline Modelo 1 & 17.07 & 4 & 4.27 & - & - & .96 & .96 & .105 \\
Modelo 2 & 19.51 & 7 & 2.79 & 2.44 & 3 & .96 & .96 & .078 \\
Modelo 3 & 20.98 & 8 & 2.62 & 3.91 & 4 & .96 & .96 & .074 \\
Modelo 4 & 30.86 & 12 & 2.57 & 13.80 & 8 & .94 & .94 & .073
\end{tabular}


Invarianza por contacto con compañero con discapacidad en clase de EF

\begin{tabular}{lcccccccc}
\hline Modelo 1 & 4.04 & 4 & 1.01 & - & - & .99 & .99 & .005 \\
Modelo 2 & 10.76 & 7 & 1.54 & 6.73 & 3 & .99 & .99 & .043 \\
Modelo 3 & 10.84 & 8 & 1.36 & 6.81 & 4 & .99 & .99 & .035 \\
Modelo 4 & 28.88 & 12 & 2.41 & $24.84^{* *}$ & 8 & .95 & .95 & .069 \\
\hline \multicolumn{7}{l}{ Invarianza por haber participado en una actividad deportiva con persona con discapacidad } \\
\hline Modelo 1 & 3.84 & 4 & .96 & - & - & .99 & .99 & .000 \\
Modelo 2 & 5.66 & 7 & .81 & 1.82 & 3 & .99 & .99 & .000 \\
Modelo 3 & 5.70 & 8 & .71 & 1.86 & 4 & .99 & .99 & .000 \\
Modelo 4 & 14.57 & 12 & 1.21 & 10.73 & 8 & .99 & .99 & .027 \\
\hline
\end{tabular}

Nota $* *=p<.01 ; * * *=p<.001 ; \mathrm{EF}=$ Educación Física

\section{Estadísticos descriptivos, correlación entre ítems, consistencia interna del instrumento y del constructo}

La Tabla 5 muestra cómo el alumnado reflejó una puntuación media global para la actitud negativa hacia la discapacidad por debajo del rango medio de la escala $(M=1.77 ; D T$ $=1.15)$. Además, se reveló una correlación positiva y moderada entre todos los ítems, mientras que la correlación entre cada ítem y la media global de actitud hacia la discapacidad en EF fue positiva en todos los casos, oscilando entre .73 y .81 $(\mathrm{p}<.001)$. Para los Indicadores de consistencia interna del instrumento y del constructo, se obtuvieron valores de $\alpha=.77 \mathrm{y}$ $\omega=.78$, respectivamente.

Tabla 5. Estadísticos Descriptivos, Alfa de Cronbach, Índice Omega y Correlaciones entre los Ítems de la EAADEF.

\begin{tabular}{lcccrrrr}
\hline Ítems-Factor & $\boldsymbol{M}$ & $\boldsymbol{D T}$ & $\mathbf{1}$ & $\mathbf{2}$ & $\mathbf{3}$ & $\mathbf{4}$ & $\mathbf{5}$ \\
\hline 1. Ítem 1 & 1.55 & .99 & & .51 & .46 & .32 & .73 \\
2. Ítem 3 & 1.72 & 1.03 & & & .56 & .43 & .81 \\
3. Ítem 5 & 1.87 & 1.03 & & & & .45 & .81 \\
4. Ítem 7 & 1.94 & 1.09 & & & & & .74 \\
5. Actitud global & 1.77 & 1.15 & & & & & \\
\hline
\end{tabular}

Nota. Todas las correlaciones fueron significativas $(\mathrm{p}<.001)$

\section{Discusión}

El objetivo de este estudio fue desarrollar y validar un instrumento para medir en el contexto español la actitud hacia el alumnado con discapacidad en EF, ofreciendo evidencias de que la EAADEF es un instrumento válido y fiable para ello. El modelo hipotetizado de dos subescalas de la actitud fue rechazado, resultando una estructura factorial unidimensional, compuesta por el componente conductual. Contar con una herramienta breve, válida y fiable permitiría una fácil aplicación en futuros programas de intervención en los que se sensibilice hacia la discapacidad. 
Para llegar al modelo unidimensional de la EAADEF fue necesario realizar cuatro AFE y un AFC. Aunque considerar el constructo con una sola dimensión no se encuentra inicialmente en línea con el modelo teórico de actitud de Triandis (1971), el modelo unidimensional obtenido en este estudio sí que coincidiría con las sugerencias del autor sobre la elaboración de escalas. En este sentido, Triandis (1971) indicó que para validar los instrumentos de medida se suelen adoptar definiciones restringidas, y como consecuencia de ello, la actitud podría ser entendida como un constructo unidimensional. Además, los resultados de este estudio sí parecen estar apoyados por un gran número de evidencias (ver revisión de Vignes et al., 2008) que han identificado esta misma problemática respecto al número de dimensiones. En esta línea, De Boer et al. (2012) proponen una concepción de la actitud bien como: un constructo único e indivisible (unidimensional), un constructo bidimensional (formado por dos componentes), o un constructo tridimensional.

Los estudios previos de Reina, Jiménez, López, García, y Hutzler (2011) y Reina et al. (2016), con una población similar en el contexto de EF, ya confirmaron las limitaciones de medida del componente afectivo de la actitud. El ATDQ (Reina et al. 2011) encontró valores de $\alpha \leq .64$ para la dimensión afectiva. Por su parte, la AISDPE (Reina et al.,

2016) es una escala con dos componentes, que no incluye el afectivo. Además, en ninguno de los dos casos se ha demostrado que la medida de la actitud sea invariante (e.g., género o contacto previo con personas con discapacidad), una de las innovaciones de este trabajo en pro de la validez de la herramienta elaborada.

Si bien evaluar la actitud solamente a través del componente cognitivo podría ser una limitación conceptual, los resultados obtenidos en el último AFE de este estudio sugieren que la EAADEF ofrece una buena medida del constructo para el contexto de EF, obteniendo una varianza explicada del $61.23 \%$, superior al $35.98 \%$ explicado por la reciente validación del CAIPE-SP al contexto español (Cordente-Mesas et al., 2016). También se podría tener en cuenta que esta limitación conceptual sería menos trascendente si se considera que en EF el componente más representativo de la actitud hacia las personas con discapacidad podría ser aquel que represente la manera de actuar o intervenir de forma práctica con los demás, es decir, el componente conductual. No obstante, dada la dificultad que se manifiesta en la bibliografía para medir la actitud hacia la discapacidad a través de un modelo tridimensional, y a que algunos autores (e.g., De Boer et al., 2012) sugieren que el mantener las dimensiones del constructo atendería más a un argumento teórico que a una sólida base empírica, es posible que el número de factores que forman un instrumento venga determinado por el contexto específico en el que la actitud sea medida. Pero ésta es una posible explicación a la variedad de herramientas que hasta la fecha existen, por lo que nuevos estudios deberían comprobar esta hipótesis. Otro posible argumento puede deberse al hecho de que Triandis no conceptualizara inicialmente el constructo de actitud para ser medido en contextos en los que se interactúe con personas con discapacidad (Bossaert y Petry, 2013). En cualquier caso, futuros trabajos deberían confirmar si para EF: primero, resultaría mejor un único modelo factorial, o podrían ser igualmente válidos y coexistir diferentes modelos para explicar la actitud (e.g., instrumentos con una, dos o/y tres dimensiones), y segundo, en el caso de resultar un modelo de dos dimensiones o más, si predominaría alguno de los componentes del constructo. 
Los resultados del análisis de consistencia interna apoyaron la validez factorial de la EAADEF. En primer lugar, se revelaron buenos valores de consistencia interna para los ítems $(\alpha)$ y para el constructo $(\omega)$ de actitud. En segundo lugar, se obtuvo una correlación moderada y positiva entre todos los ítems y la medida global de actitud. En tercer lugar, y posiblemente uno de los resultados más prometedores, se obtuvieron mejores pesos de regresión para los ítems de la EAADEF con respecto a la AISDPE, que parecen dotar de mayor calidad la medida del constructo (i.e., pesos de regresión de la EAADEF $\geq .57$, mientras que de la AISDPE fueron $\geq$ .31). Futuros trabajos podrían analizar si la actitud que muestran los estudiantes se mantiene estable a lo largo del tiempo, analizando así la estabilidad temporal de la EAADEF.

Los resultados del análisis de invarianza muestran que la EAADEF puede ser administrada para chicos y chicas, así como entre el alumnado que ha mantenido o no contacto con: familiar o amigo con discapacidad, estudiante con discapacidad en clase de EF, y/o persona con discapacidad en una actividad deportiva. Estudios previos han relacionado la actitud hacia la discapacidad en EF con variables como el género o el contacto previo con una persona con discapacidad (Campos et al., 2014; Reina et al., 2011, 2016). Sin embargo, la mayoría de los instrumentos utilizados hasta la fecha, como el CAIPE-R (Campos, Ferreira, y Block, 2013), el CAIPE-SP (Cordente-Mesas et al., 2016), el CATCH (Rosenbaum, Armstrong, y King, 1986) o el AISDPE (Reina et al., 2016), no han ofrecido evidencias de que fuera posible establecer tales relaciones. Así, los resultados de invarianza aquí presentados permiten comparar las puntuaciones medias obtenidas de la EAADEF con las cuatro variables socio- demográficas analizadas. Esta aportación puede ser interesante para realizar programas de intervención con grupos experimentales, manipulando algunas de estas variables socio- demográficas.

Aunque todavía son necesarias futuras investigaciones que confirmen la estructura factorial de la actitud para el contexto escolar, los resultados obtenidos sugieren que la EAADEF puede ser un instrumento válido y fiable para medir la actitud hacia el alumnado con discapacidad en EF en el contexto español. Esta nueva escala es una herramienta breve y rápida de administrar que: i) ayudaría a los investigadores a identificar los antecedentes y consecuencias de las actitudes hacia la inclusión de alumnado con discapacidad en EF; y ii) ofrecería a los docentes de EF una herramienta que permite evaluar la puesta en práctica de una metodología inclusiva.

\section{Referencias bibliográficas}

Aguado, A., Alcedo, M. A., y Arias, B. (2008). Cambio de actitudes hacia la discapacidad con escolares de primaria. Psicothema, 20(4), 697-704.

American Psychological Association (2010). Publication Manual of the American Psychological Association ( $6^{\mathrm{a}}$ ed.). Washington, DC: American Psychological Association.

Block, M. E. (1995). Development and validation of the children's attitudes towards integrated physical education-revised. Adapted Physical Activity Quarterly, 12, 60-70.

Bossaert, G., y Petry, K. (2013). Factorial validity of the Chedoke-McMaster Attitudes towards Children with Handicaps Scale (CATCH). Research in Developmental Disabilities, 34, 1336-1345. doi: 10.1016/j.ridd.2013.01.007.

Brown, T. A. (2006). Confirmatory factor analysis for applied research. Nueva York: The Guilford Press. 
Byrne, B. M. (2001). Structural equation modelling with Amos: Basic concepts, applications, and programming. Mahwah, NJ: Erlbaum.

Byrne, B. M., Shavelson, R. J., y Muthén, B. (1989). Testing for the equivalence of factor covariance and means structures: The issue of partial measurement invariance. Psychological Bulletin, 105, 456-66. doi:10.1037//0033-2909.105.3.456.

Campos, M., Ferreira, J., y Block, M. (2013). An analysis into the structure, validity and reliability of the children's attitudes towards integrated physical education revised (CAIPE-R). European Journal of Adapted Physical Activity, 6(2), 29-37.

Campos, M., Ferreira, J., y Block, M. E. (2014). Influence of an awareness program on Portuguese middle and high school students' perceptions of peers with disabilities. Psychological Reports, 115(3), 897-912. doi: 10.2466/11.15.PR0.115c26z7.

Cordente-Mesas, D., González-Víllora, S., Block, M. E., y Contreras-Jordán, O. R. (2016). Structure, validity and reliability of the Children' $\mathrm{s}$ Attitudes towards Integrated Physical Education-Spanish version (CAIPE-SP). European Journal of Adapted Physical Activity, 9(2), 3-12.

De Boer, A., Timmerman, M. E., Pijl, S. J., y Minnaert, A. (2012). The psychometric evaluation of a questionnaire to measure attitudes towards inclusive education. European Journal of Psychology of Education, 27, 573-89. doi:10.1007/s10212-011-0096-z.

Flórez, M. A., Aguado, A. L., y Alcedo, M. A. (2009). Revisión y análisis de los programas de cambio de actitudes hacia personas con discapacidad. Anuario de Psicología Clínica y de la Salud, 5, 85-98.

Hu, L., y Bentler, P. M. (1999). Cut-off criteria for fit indexes in covariance structure analysis: Conventional criteria versus new alternatives. Structural Equation Modelling, 6, 155. doi: 10.1080/10705519909540118.

Jöreskog, K. G., y Sörbom, D. (1993). LISREL 8: Structural equation modelling with the SIMPLIS command language. Chicago: Scientific Software.

Lindsay, S., y Edwards, A. (2013). A systematic review of disability awareness interventions for hildren and youth. Disability and Rehabilitation, 35(8), 623-646. doi:10.3109/09638288.2012.702850.

Nunnally, J. C. (1978). Psychometric theory (2nd ed.). New York: McGraw-Hill.

Ocete, C. (2016). "Deporte inclusivo en la escuela": diseño y análisis de un programa de intervención para promover la inclusión del alumnado con discapacidad en educación física (Tesis doctoral). Extraído desde: https://www.educacion.gob.es/teseo/ mostrarRef.do?ref=1200945.

Reina, R., Hutzler, Y., Iniguez-Santiago, M. C., y Moreno-Murcia, J. A. (2016). Attitudes towards inclusion of students with disabilities in physical education questionnaire (AISDPE): A two component scale in Spanish. European Journal of Human Movement, 36, 75-87.

Reina, R., López, V., Jiménez, M., García, T., y Hutzler, Y. (2011). Effects of awareness Interventions on children's attitudes towardpeers withavisual impairment. International Journalof RehabilitationResearch,34(3),235-42.doi:10.1097/MRR.0b013e3283487f49.

Rosenbaum, P., Armstrong, R., y King S. (1986). Children's attitudes toward disabled peers: a self-report measure. Journal of Paediatric Psychology, 11, 517-30. 
Schermelleh-Engel, K., Moosbrugger, H., y Müller, H. (2003). Evaluating the fit of structural equation models: tests of significance and descriptive goodness-of-fit measures. Methods of Psychological Research Online, 8(2), 23-74.

Triandis, H. C. (1971). Attitude and attitude change. New York: Wiley.

Vignes, C., Coley, N., Grandjean, H., Godeau, E., y Arnaud, C. (2008). Measuring children's attitudes towards peers with disabilities: a review of instruments. Developmental Medicine and Child Neurology, 50(3), 182-89. doi:10.1080/1034912X.2012.723944.

\section{Anexo}

\section{Escala de Actitud hacia el Alumnado con Discapacidad en Educación Física (EAADEF)}

1. Prefiero no relacionarme con personas con discapacidad

3. Evitaría hacer un trabajo de clase con una persona con discapacidad

5. Evitaría para mi equipo a una persona con discapacidad

7. No propondría como capitán de mi equipo a una persona con discapacidad

Nota. Ítems eliminados del componente cognitivo: 2. Las personas con discapacidad no pueden realizar la mayoría de los deportes practicados por personas sin discapacidad. 4. Los deportes adaptados deben tener una estructura sencilla y con pocas reglas para facilitar la participación de personas con discapacidad. 6. Pienso que destacaría en una actividad física o deportiva si participo con personas con discapacidad. 8. Las personas con ceguera siempre han de llevar un guía que los acompañe de un lado par. 